\title{
Dificultades para la colaboración en investigación entre cardiólogos y médicos de urgencias
}

\section{Ò Miró}

\section{Sr. Director:}

A raíz de mi artículo previamente publicado en Anales del Sistema Sanitario de Navarra acerca de la necesidad de intensificar la colaboración en investigación entre cardiólogos y médicos de urgencias ${ }^{1}$, García del Águila y col realizan un interesante comentario sobre el papel que deben tener los sistemas de emergencias médicas (SEM) en la investigación española en general y, particularmente, en los procesos cardiovasculares urgentes ${ }^{2}$. Comparto con los autores la necesidad de que los SEM se integren en la investigación multidisciplinar, que es la tendencia que se ha impuesto durante los últimos años siguiendo una lógica irrebatible: el enfermo y su enfermedad son una única entidad que centra el proceso asistencial, y son los profesionales que participan de este proceso los que deben aunar y coordinar esfuerzos en torno a él. Experiencias en otros países nos enseñan que es posible ${ }^{3}$. El síndrome coronario agudo (SCA), en cualquiera de sus expresiones, constituye el ejemplo paradigmático de lo bien que han llegado a coordinarse los profesionales para ofrecer una respuesta asistencial adecuada por el bien del paciente 4 . Toca pues ahora poner en práctica en el terreno de la investigación esas mismas complicidades que han permitido una asistencia tan coordinada. No existe receta mágica, pero unas dosis de

Área de urgencias. Hospital Clínic. Barcelona. generosidad por una parte, de esfuerzo en formación por otra y el convencimiento de todos de que esta colaboración es necesaria deben ayudar a hacerlo posible. Artículos recientemente publicados demuestran que los SEM españoles ya están llevando a cabo una actividad investigadora relevante en múltiples campos de la patología cardiovascular ${ }^{5-10}$, y algunos de estos trabajos, realizados en colaboración con otros especialistas, han sido firmados por alguno de los autores de la carta ${ }^{8-10}$.

Por su parte, Alquézar Arbé y col $^{11}$ apuntan a que estas dificultades de colaboración no son abordadas suficientemente desde las sociedades científicas, focalizando su comentario en la Sociedad Española de Medicina de Urgencias y Emergencias (SEMES). Resaltan, y con razón, que en los grupos de trabajo relativos a patología cardiovascular de dicha sociedad no se encuentran cardiólogos entre sus miembros. Esta es una situación recíproca, y hasta diría que ubicua en España, y que sin duda debiera solventarse. Algunas iniciativas se están produciendo en este sentido, entre las que cabe destacar la publicación de guías de consenso entre sociedades de distintas especialidades (las cuales, ciertamente, no entrañan investigación propiamente dicha) ${ }^{12,13}$. Tal vez una iniciativa que merece una mención especial sea la del GREAT (Global Research in Acute Con- 
ditions Team). Se trata de un grupo multidisciplinar internacional que engloba, con finalidades investigadoras, especialistas tan diversos como cardiólogos, intensivistas, internistas o médicos de urgencias ${ }^{14} \mathrm{y}$ en el cual existe representación española de especialistas en muchos de estos campos. El grupo ha producido en los últimos 3 años una docena de documentos, algunos publicados en revistas de primer nivel ${ }^{15,16}$. Tal vez una iniciativa similar, de ámbito español, podría ayudar a romper estos viejos esquemas individualistas desde la perspectiva de las especialidades. Finalmente, comparto con los autores que el punto de vista de cada una de las especialidades para una patología concreta pueden ser diferentes, así como sus intereses en investigación. Tal es el caso que los autores citan con respecto a los biomarcadores ${ }^{17-19}$, pero también podría extenderse esta disparidad a otras exploraciones complementarias en cardiología ${ }^{20,21} \mathrm{o}$ a pacientes con formas de presentación determinadas de una patología en la cual puede existir mayor interés en algún colectivo de especialistas respecto a otros ${ }^{22}$. Sin duda, el poner en común la pregunta de investigación entre todos los intervinientes en la asistencia enriquecerá los resultados de dicha investigación, y mi artículo pretendía ser una llamada a esta colaboración ${ }^{1}$.

\section{BIBLIOGRAFÍA}

1. Miró O. Necesidad de colaboración en investigación entre cardiólogos y médicos de urgencias. An Sist Sanit Navar 2014; 37: 9-16

2. García del Águila J, Mellado Vergel FJ, Rosell ORTIZ F. La colaboración en investigación entre cardiólogos y médicos de urgencias requiere un planteamiento nuevo. An Sist Sanit Navar 2014; 37:

3. Farzi S, Hausler F, Wallner S, Spindelböck W, Prause G, Gemes G. Medición prehospitalaria del exceso de base arterial y su posible papel en la predicción del desenlace tras una parada cardiaca extrahospitalaria. Emergencias 2013; 25: 47-50.

4. Gómez-Hospital JA, Domenico Dallaglio P, Sánchez-Salado JC, Ariza A, Homs S, Lorente V et al. Impact on delay times and characteristics of patients undergoing primary percutaneous coronary intervention in the southern metropolitan area of Barcelona after implementation of the infarction code program. Rev Esp Cardiol 2012; 65: 911-918.

5. López-Messa JB, Alonso-Fernández JI, Andrés De Llano JM, Garmendia-Leiza JR, Ardura-Fernández J, De Castro-Rodríguez F et al. Características generales de la parada cardiaca extrahospitalaria registrada por un servicio de emergencias médicas. Emergencias 2012; 24: 2834.

6. Barreña Oceja I, Gil Martín FJ, García De Vicuña Meléndez A, Rodríguez Delgadillo MA, GutiérRez Herrador G, Vázquez Naveira MP. Resultados de la puesta en marcha de un protocolo de hipotermia terapéutica en la parada cardiaca consensuado entre un sistema de emergencias médicas y un servicio de urgencias hospitalario. Emergencias 2012; 24: 39-43.

7. Corral Torres E, Fernández Avilés F, López De Sa Areses E, Martín Benítez JC, Montejo JC, MarTín REYEs R et al. La aplicación de hipotermia moderada tras la reanimación cardiaca iniciada en el medio extrahospitalario puede incrementar la supervivencia sin deterioro neurológico. Estudio de casos y controles. Emergencias 2012; 24: 7-12.

8. Armstrong PW, Gershlick AH, Goldstein P, WiLCOX R, DANAYS T, LAMBert Y, et al. Fibrinolysis or primary PCI in ST-segment elevation myocardial infarction. N Engl J Med 2013; 368: 1379-1387.

9. Bolívar Muñoz J, Martínez Cassinello R, Mateo Rodríguez I, Torres Ruz JM, Pascual Martínez $\mathrm{N}$, Rosell Ortiz F et al. Actuación de los pacientes ante un síndrome coronario agudo: diferencias desde una perspectiva de género. Emergencias 2013; 25: 23-30.

10. Rosell Ortiz F, Mellado Vegel F, Fernández Valle P, González lobato I, Martínez Lara M, Ruiz MONTERo MM et al. Descripción y resultados iniciales del registro andaluz de parada cardiaca extrahospitalaria. Emergencias 2013; 25: 345-352.

11. Alquézar Arbé A, Serés Roig M, Higa Sansone M, BláZqueZ Andión M. Dificultades para la colaboración en investigación entre cardiólogos y médicos de urgencias. An Sist Sanit Navar 2014; 37:

12. Martín A, Merino JL, del Arco C, Martínez Alday J, Laguna P, ArRibas F et al. Documento de consenso sobre el tratamiento de la fibrilación auricular en los servicios de urgencias hospitalarios. Rev Esp Cardiol 2003; 56: 801-16.

13. Martín Martínez A, Fernández Lozano I, CollVinent Puig B, Tercedor Sánchez L, Del Arco Galán C, Arribas Ynsaurriaga F et al. Manejo de 
los pacientes con fibrilación auricular en los servicios de urgencias hospitalarios (actualización 2012). Emergencias 2012; 24: 300-324.

14. GREAT (Global Research in Acute Conditions Team). Accedido: 13/10/2014. Accesible en: http://www.greatnetwork.org/int/

15. Mebazaa A, Gayat E, Lassus J, Meas T, Mueller C, MAGgioni A, et al. Association between elevated blood glucose and outcome in acute heart failure: results from an international observational cohort. J Am Coll Cardiol 2013; 61: 820-829.

16. Shah R, Gayat E, Januzzi JL JR, Sato N, CohenSolal A, DISomma $\mathrm{S}$ et al. Body mass index and mortality in acutely decompensated heart failure across the world: a global obesity paradox. J Am Coll Cardiol 2014; 63: 778-785.

17. Marino Genicio R, García García A, Prieto García B, Rodríguez Casado V, Jacob Rodríguez J, Herrero Puente P. Utilidad de la MR-proadrenomedulina y de la copeptina como predictores de mortalidad a los 30 días en pacientes con insuficiencia cardiaca aguda. Emergencias 2013; 25: 328-329.

18. Rubini M, López B, Rubini S, Mueller C. Biomarcadores en el paciente con dolor torácico: pasado, presente y futuro. Emergencias 2014; 26: 221-226.

19. Miró O, Jacob J, Martín-SÁnchez FJ, Herrero P, PAvón J, PÉREz-DurÁ MJ et al. Implicaciones pronósticas de la posibilidad de determinar con carácter urgente el péptido natriurético tipo B en el servicio de urgencias en pacientes con insuficiencia cardiaca aguda: estudio PICASU-2. Emergencias 2011; 23: 437-446.

20. Mas-Stachurska A, Miró O, Sitges M, de Caralt TM, PEREA RJ, López B et al. Exercise echocardiography and multidetector computed tomography for the evaluation of acute chest pain. Rev Esp Cardiol 2014; en prensa (doi: 10.1016/j.recesp.2014.05.009).

21. Gil Román JJ, González Méndez A, Alonso Morilla A, Irimia A, Maujo Fernández J, Herrero PuenTE P. Factores asociados con una ergometría positiva en pacientes con dolor torácico y riesgo bajo-moderado de síndrome coronario agudo. Emergencias 2013; 25: 327-328.

22. Sánchez M, Vázquez J, Temboury R, Capdepon C, Povar J, Santaló M. El síndrome coronario agudo inclasificable en los servicios de urgencias españoles. Aportaciones del registro MUSICA. Emergencias 2013; 25: 263-267. 